\title{
Decreased heart rate variability in children born with low birth weight
}

\author{
Alexander Rakow' ${ }^{1}$ Miriam Katz-Salamon', Mats Ericson ${ }^{2}$, Ann Edner ${ }^{1}$ and Mireille Vanpée ${ }^{1}$
}

BACKGROUND: Low birth weight (LBW) is associated with cardiovascular morbidity in adulthood. Imbalance in the autonomic nervous system (ANS) has been implicated as a mechanism behind the developmental programming of cardiovascular function. We hypothesized that deviations in the ANS function are seen in children born with LBW.

METHODS: Eighty-six children were included: 31 born preterm (<32 wk gestational age), 27 born at term but small for gestational age (SGA), and 28 born at term with normal birth weight (control). Twenty-four-hour Holter-electrocardiogram monitoring was performed at an average age of $9 \mathrm{y}$. Heart rate variability results were analyzed using frequency and time domain methods.

RESULTS: All frequency components and both time domain parameters tested were significantly lower in the preterm and SGA children compared with controls. The low frequency/high frequency ratio was not significantly different between children born with LBW and controls.

CONCLUSION: The autonomic control appears to be affected in children born with LBW despite gestational age at birth. Decreased total power, as an estimation of the ANS's global activity, rather than the balance between parasympathetic and sympathetic modulation might be an early marker of cardiovascular disease later on in life for LBW born children.

L ow birth weight (LBW) might have an impact on health by increasing the risk for hypertension, cardiovascular disease (CVD), diabetes mellitus, asthma, and allergies as well as by influencing cognitive function and behavior (1-4). The autonomic nervous system (ANS), with its balancing actions of the sympathetic and parasympathetic branches, regulates cardiovascular function starting during fetal life. If this equilibrium is impaired, development of the above-mentioned disease states could be possible (5).

Studies on control of heart rhythm are based on estimations of changes in the heart rate (HR) referred to as HR variability (HRV). HRV represents the variation over time of the period between consecutive heartbeats modified by the sympathetic and parasympathetic nervous system (6). HRV is thought to reflect the heart's ability to adapt to changing circumstances by detecting and quickly responding to unpredictable stimuli
(6). As such, HRV provides a noninvasive evaluation of the changes in HR in different physiological situations; it can also be used as a predictor of cardiac dysfunctions (7). The analysis of HRV was standardized in 1996 by the Task Force of the European Society of Cardiology and the North American Society of Pacing Electrophysiology and has become a wellestablished tool for evaluating ANS function (6).

There are several methods for analyzing HRV. In this study, we use time and frequency domain analysis (6). The power spectrum was divided into four different frequency bands: total power, as an estimation of the ANS's global activity (Tot Pow: $0.0033-0.40 \mathrm{~Hz}\left(\mathrm{~ms}^{2}\right)$ ); very low frequency power, representing mainly parasympathetic activity (VLF Pow: $0.0033-0.04$ $\mathrm{Hz}\left(\mathrm{ms}^{2}\right)$ ); low frequency power, jointly mediated by parasympathetic and ( $\beta$-) sympathetic activities (LF Pow: 0.04-0.15 $\left.\mathrm{Hz}\left(\mathrm{ms}^{2}\right)\right)$; and high frequency power, representing parasympathetic activity (HF Pow: $0.15-0.40 \mathrm{~Hz}\left(\mathrm{~ms}^{2}\right)$ ). The ratio of LF Pow to HF Pow is regarded to be a marker for the ANS balance. In the time domain, the mean of the SD of all normal $R$ wave to $R$ wave (RR) intervals for all 5 min segments of each electrocardiogram (ECG) recording (SD of normal to normal RR intervals (SDNN) index) was calculated (6).

An imbalance of the ANS with a shift of autonomic control towards sympathetic predominance is regarded as a predictor for poor outcome in adult patients with previous myocardial infarction $(7,8)$. A prospective study has shown that reduced HRV was associated with sudden death in patients with previous myocardial infarction and ventricular arrhythmia (9). Moreover, it is suggested that reduced HRV is associated with higher mortality even among individuals free of CVD (10). Environmental factors such as overweight, obesity, smoking, or fetal exposure to smoking may also affect ANS function in adults and infants (11-13).

In a previous study we found that children born preterm (PT) or at term but small for gestational age (SGA) had higher $\mathrm{HR}$ at rest, and during and after a mathematical stress test, and had higher urinary catecholamines as compared with children born at term with normal birth weight (14). These findings suggested an increased sympathetic tone in children born with LBW. In this study, we hypothesize that young children born with LBW have measurable alterations in autonomic function 
and that HRV measurements might be useful for early detection of individuals at risk for the development of CVD.

\section{RESULTS}

\section{Clinical Characteristics}

Maternal, perinatal data and characteristics at the time of the visit are shown in Table 1. Maternal risk factors were not significantly different between the groups. Children born PT were younger, shorter, and lighter than controls. Children born SGA were lighter and shorter than controls and had a lower BMI (Table 1).

\section{Time and Frequency Domain Measurements of HRV}

All frequency domain parameters (Tot Pow, HF Pow, VLF Pow, and LF Pow) were significantly lower in the children born PT or SGA than in controls $(P=0.008-0.04)$, whereas the LF Pow/ HF Pow ratio was similar in all children $(P=0.09)$. Mean RR and the SDNN was significantly lower in the groups of children born PT or SGA than in controls $(P=0.02 ; 0.006$, respectively), (Figure 1; Table 2).

Ten control, five PT, and three SGA children fulfilled the criteria for overweight and obesity $\left(\mathrm{BMI}>20 \mathrm{~kg} / \mathrm{m}^{2}\right)$. After exclusion of these children, the differences between children born with LBW (PT and SGA) and controls became more

Table 1. Subject characteristics for healthy children born PT, born at term but SGA, or born at term with normal birth weight

\begin{tabular}{|c|c|c|c|c|}
\hline & $\begin{array}{c}\text { PT } \\
(n=31)\end{array}$ & $\begin{array}{c}\text { SGA } \\
(n=27)\end{array}$ & $\begin{array}{l}\text { Control } \\
(n=28)\end{array}$ & $P$ value \\
\hline \multicolumn{5}{|l|}{ Maternal data } \\
\hline Smoking, $n(\%)$ & $6(19.3)$ & $6(22.2)$ & $6(21.4)$ & 0.96 \\
\hline $\begin{array}{l}\text { Gestational } \\
\text { diabetes, } n(\%)\end{array}$ & $0(0)$ & $2(7.4)$ & $0(0)$ & 0.1 \\
\hline $\begin{array}{l}\text { Prenatal steroids, } \\
n(\%)\end{array}$ & $2(7.4)$ & $0(0)$ & $0(0)$ & 0.13 \\
\hline $\begin{array}{l}\text { Gestational } \\
\text { hypertension, } \\
n(\%)\end{array}$ & $5(16.6)$ & $3(11.5)$ & $0(0)$ & 0.08 \\
\hline $\begin{array}{l}\text { History of } \\
\text { hypertension, } \\
n(\%)\end{array}$ & $3(10.3)$ & $1(3.8)$ & $0(0)$ & 0.18 \\
\hline \multicolumn{5}{|l|}{ Perinatal data } \\
\hline Girls, $n(\%)$ & $15(48.4)$ & $16(59.3)$ & $18(64.3)$ & 0.44 \\
\hline Birth weight, $g$ & $965(202)^{*}$ & $2,441(334)^{*}$ & $3,503(515)$ & $<0.0001$ \\
\hline $\begin{array}{l}\text { Gestational age, } \\
\text { weeks }\end{array}$ & $26.7(2.1)^{*}$ & $39.3(1.4)$ & $39.6(1.0)$ & $<0.0001$ \\
\hline \multicolumn{5}{|c|}{ Characteristics at visit } \\
\hline Age, years & $9.6(0.3)^{*}$ & $9.8(0.3)$ & $9.8(0.2)$ & 0.007 \\
\hline Weight, kg & $31.8(7.1)^{*}$ & $31.8(7.3)^{*}$ & $37.9(8.1)$ & 0.003 \\
\hline Height, cm & $134.2(6.8)^{*}$ & $137.4(7.3)^{*}$ & $142.6(7.5)$ & 0.0003 \\
\hline $\mathrm{BMI}, \mathrm{kg} / \mathrm{m}^{2}$ & $17.6(2.9)$ & $16.8(2.5)^{*}$ & $18.6(2.8)$ & 0.04 \\
\hline \multicolumn{5}{|c|}{ Values are mean (SD) or numbers, $n(\%)$. } \\
\hline \multicolumn{5}{|c|}{$\begin{array}{l}{ }^{*} P<0.05 \text { for indicated group vs. the control group, according to Kruskal-Wallis test or } \chi^{2} \text {, } \\
\text { according to Pearson. }\end{array}$} \\
\hline \multicolumn{5}{|c|}{$\begin{array}{l}\text { Control, born at term with normal weight for gestational age; PT, preterm; SGA, small for } \\
\text { gestational age. }\end{array}$} \\
\hline
\end{tabular}

pronounced (Tot Pow: $P=0.002$; HF Pow: $P=0.002$; VLF Pow: $P=0.01$; LF Pow: $P=0.01$; LF Pow/HF Pow ratio: $P=0.054$; mean RR: $P=0.01$; SDNN: $P=0.002$ ).

No cerebral palsy or severe cognitive impairment was diagnosed during the neonatal follow-up or at the time of investigation, and all children attended normal schools. Five PT children and one SGA child had minor neurological symptoms. The re-analysis of the data after exclusion of the six patients with minor neurological symptoms did not affect the results (data not shown).

Logistic regression analysis was done for all HRV parameters where independent variables had F-to-enter $=4$. Subject and maternal characteristics did not show significant dependence to HRV parameters.

\section{DISCUSSION}

Our findings demonstrate that LBW, whether due to PT delivery or intrauterine growth retardation, affects ANS function in childhood when measured between 9 and $10 \mathrm{y}$ of age. The significantly lower values for all power spectra and both time domain parameters suggest decreased overall activity of the ANS among the PT and SGA groups. These results indicate a less active and less reactive ANS in children born with LBW, implicating an impaired ability to adapt to ongoing changes in the internal and external milieu.

The biological relevance of decreased HRV is well documented in patients after acute myocardial infarction and some noncardiovascular morbidities $(7,10)$. It is not yet known whether changes in the function of ANS could potentially play a role in the later development of hypertension and/or cardiovascular morbidity in children born with LBW owing to prematurity and/or SGA.

The strength of our study is the well-defined groups of children born with LBW (PT and SGA), allowing us to infer differences between fetal stress in SGA or postnatal stress in premature infants. Gestational age (mean: $27 \mathrm{wk}$ ) and birth weight (mean: $1,000 \mathrm{~g}$ ) in the group of children born PT well represents today's neonatal population in the Western world.

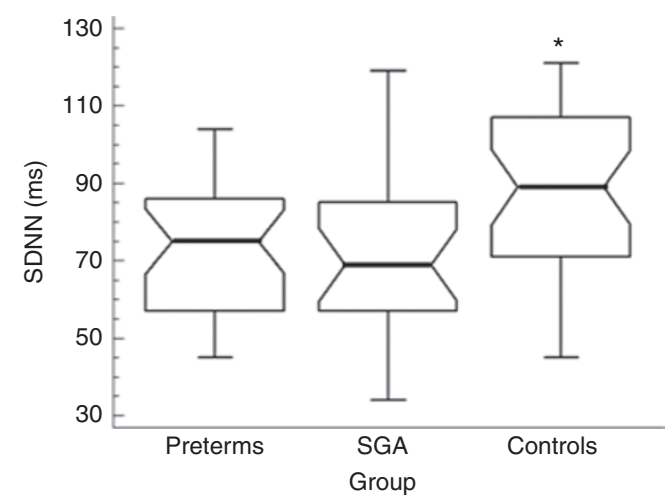

Figure 1. SD of all normal to normal $R$ wave intervals (SDNN) $\left(\mathrm{ms}^{2}\right)$ in healthy children born preterm (PT), born at term but small for gestational age (SGA), or born at term with normal birth weight (controls). Values are shown as a box-plot with the medians indicated as bold horizontal lines. The bars extend out to the minimum and maximum values. ${ }^{*} P=0.006$ for indicated group vs. the control group, according to Kruskal-Wallis test. 
Table 2. Heart rate variability parameters in time and frequency domains for healthy children born PT, born at term but SGA, and born at term with normal birth weight

\begin{tabular}{|c|c|c|c|c|}
\hline & PT $(n=31)$ & $\mathrm{SGA}(n=27)$ & Control $(n=28)$ & $P$ value \\
\hline Tot Pow, $\mathrm{ms}^{2}$ & $4,405^{*}(3,514-5,297)$ & $3,965^{*}(3,030-4,901)$ & $6,344(5,109-7,579)$ & 0.008 \\
\hline HF Pow, $\mathrm{ms}^{2}$ & $1,815^{*}(1,299-2,331)$ & $1,363^{*}(994-1,732)$ & $2,934(2,099-3,769)$ & 0.01 \\
\hline LF Pow, $\mathrm{ms}^{2}$ & $1,429^{*}(1,132-1,744)$ & $1,399 *(1,036-1,738)$ & $1,875(1,509-2,074)$ & 0.04 \\
\hline $\mathrm{LF} / \mathrm{HF}$ ratio & $1.4(1.2-1.6)$ & $1.6(1.4-1.8)$ & $1.4(1.1-1.7)$ & 0.09 \\
\hline SDNN, ms & $74^{*}(67-81)$ & $71 *(63-79)$ & $89(80-98)$ & 0.006 \\
\hline
\end{tabular}

Values are means and $95 \%$ confidence intervals.

*P $\leq 0.05$ for indicated group vs. the control group, according to Kruskal-Wallis test.

Control, born at term with normal weight for gestational age; HF Pow, high frequency power; LF Pow, low frequency power; LF/HF ratio, low frequency/high frequency ratio; mean RR, mean of R wave to R wave variation; PT, preterm; SDNN, the mean of the SD of all normal RR intervals for all 5-min segments; SGA, small for gestational age; Tot Pow, total power; VLF Pow, very low frequency power.

In a recent, similar study (15), children born at term with LBW owing to intrauterine growth retardation and children born at term with appropriate birth weight were investigated at the age of 9-10 y using HRV measurements. When groups were compared, no differences could be found regarding HRV measurements; it was only when the group of intrauterine growth retardation children was subdivided into those $<2.5 \mathrm{~kg}$ and those $>2.5 \mathrm{~kg}$ that those born with birth weight below $2.5 \mathrm{~kg}$ were significantly different from controls and from the intrauterine growth retardation group with higher weight at birth.

In PT infants at term age ( $40 \mathrm{wk}) \mathrm{HRV}$ studies revealed a delayed development of the ANS function (16). However, when the same subjects were re-investigated at $2-3$ and 6-7 y, no significant difference in HRV parameters persisted in comparison with the controls (16). The impairment in ANS function among the group of premature infants was suggested to be an effect of stress owing to pain, noise, visual stimuli, inadequate nutrition, and ventilator support (17-19). It is known that the sympathetic nervous system matures earlier in fetal life than the parasympathetic nervous system but then becomes increasingly outbalanced beyond $30 \mathrm{wk}$ of gestational age, which could explain a temporary dominance of the sympathetic system at the beginning of life for children born PT $(19,20)$. Even in children born SGA, higher sympathetic tone and lower vagal tone has been observed postnatal at 1 and 3 mo of age (5). However, our findings demonstrated that the deviations in heart rhythm control are long lasting and present at 9-10 y of age. This is in line with studies on adults born at term but SGA and with a final short height at $22 \mathrm{y}$ of age. Those individuals were shown to have increased sympathetic nerve traffic measured by muscle sympathetic nerve activity (21). Therefore, the results are partly in concordance with our findings showing a less-pronounced influence of the parasympathetic branch (decreased HF Pow) and thereby a sympathetic predominance in the function of the ANS in children born SGA at term. In this study, we do not detect any significant difference between PT and SGA born children regarding HRV parameters, but both groups differ significantly from the control group, which probably allows the speculation that the pathogenesis for LBW is secondary and the key factor is LBW itself.
Maternal smoking during pregnancy, obesity, and overweight are factors that downregulate $\operatorname{HRV}(12,13)$. In the present study, the prevalence of mothers smoking was similar in all groups tested. In our cohort 10 children from the control group, five children from the PT group and three children from the SGA group fulfilled the criteria for overweight and obesity, which correlates well to the increasing problem of overweight among children in Sweden (22). After exclusion of children with BMI $>20$ from all three groups, the differences in HRV parameters between children born with LBW and controls were even more pronounced.

Children diagnosed with central coordination disturbances have demonstrated lower values for HRV parameters (23). Therefore it has been suggested that HRV measurements could be used as a prognostic tool in the diagnosis of cerebral palsy in infants (23). The results from the present study do not show the similar association between disturbances in motor- and cardiovascular control because the exclusion of the six patients with minor neurological symptoms did not affect the results. However, no cerebral palsy or severe cognitive impairments were diagnosed during the neonatal follow-up or at the time of investigation in our study population, and all children attended normal schools.

To our knowledge, this is the first study demonstrating the long-lasting impairment in ANS regulation of heart rhythm in children born with LBW. An imbalance of the ANS with a relatively increased sympathetic tone might be one of the causative mechanisms for the increased risk of developing metabolic disorders and CVD in the population of individuals born with LBW. However, in the present study a general decreased activity of the ANS is shown for both groups (PT and SGA) with significantly lower values for Tot Pow, HF Pow, LF Pow, VLF Pow, SDNN RR, and mean RR as compared with the controls. Our data do not support any dominance of the sympathetic nervous system over the parasympathetic branch of the ANS. The ratio between LF Pow and HF Pow as an indicator for the balance of the ANS is not different for our study groups. It is still under debate whether the LF Pow/HF Pow ratio is a reliable measurement for the ANS balance or rather only an approximation (24). As previously shown 
by our group with the same cohort, children born with LBW had significantly higher HR and urinary catecholamines (14). Whether this is due to the overall suppression of ANS activity is not entirely conclusive and needs further investigation.

We speculate that an overall suppression of the ANS, as shown in the present study, could be an indicator for an impaired capacity of the investigated groups of LBW born children to adequately adapt to changing circumstances and that this could be a contributing factor for the later development of hypertension and CVD.

\section{METHODS}

\section{Study Design and Population}

All children were singletons born without chromosomal anomalies, congenital infections or life threatening congenital anomalies at Karolinska University Hospital between 1990 and 1993. Eligible infants were consecutively born infants $<32$ wk gestational age (PT) admitted to the neonatal intensive care unit, or full-term infants born SGA and admitted to the maternity ward. After inclusion of PT and SGA children, children born at term with normal birth weight (controls), and matched for date of birth and sex, were selected from the delivery room records. All control infants were healthy at birth and had not been admitted to the neonatal intensive care unit. A total of 390 families were eligible, but 133 were lost to follow-up owing to changed addresses. Of the remaining 257 families invited, 105 agreed to participate in the study. Twenty-four-hour ECG recordings were successfully obtained in 92 children. In six children, the recordings could not be used owing to poor technical quality. Eighty-six children with successful 24-h ECG recordings were included: 31 PT children, 27 SGA children, and 28 control children (Table 1).

Gestational age was determined by ultrasound in early pregnancy (25). Term was defined as a gestational age between 37 and $42 \mathrm{wk}$. SGA was defined as a birth weight $<$ mean -2 SD (i.e., below the sex specific 2.5 th percentile for gestational age) according to Swedish reference data for normal fetal growth (26). Of the 31 children in the PT group, only one child was SGA. Of the 27 children in the SGA group, six had a ponderal index $<2.3$, suggesting asymmetrical intrauterine growth. Ponderal index was calculated by birth weight $(\mathrm{kg}) \times 100 /$ birth length $\left(\mathrm{cm}^{3}\right)(27)$.

Information on maternal and neonatal characteristics was collected from the Swedish birth records. Maternal age, maternal smoking, and maternal history of hypertension/preeclampsia, prenatal steroid administration, date of birth, gestational age, and birth weight were noted (Table 1).

Anthropometric data (weight and height) were obtained in all children by the same research nurse. BMI was calculated by the formula: weight $(\mathrm{kg}) /$ height $\left(\mathrm{m}^{2}\right)$. Overweight and obesity were defined using the International Obesity Task Force age- and sex-specific cut-off criteria $(22,28)$. A medical history was obtained and a physical examination was performed by the same physician for $95 \%$ of the children. All children were in good health at the time of investigation at an average age of $9.7(0.3 \mathrm{SD}) \mathrm{y}$. Five children in the PT group and one child in the SGA group had minor neurological findings. No child suffered from severe cerebral palsy or cognitive impairments. All children attended normal schools. At the time of investigation, a total number of seven children had started puberty at low Tanner stages (29). One child in the PT group and two children in the control group had entered puberty at Tanner stage 2. One child from the SGA group and one from the control group were between Tanner stage 2 and 3 and one child from the control and one from the SGA group were at Tanner stage 3.

The study was approved by the Ethical Committee at Karolinska University Hospital. Written and oral consent was obtained from the parents and children.

\section{Physiological Measurements}

Holter-ECG was obtained for $24 \mathrm{~h}$ in 86 children using an ambulatory recorder unit (Braemer DL 700; Braemer, Burnsville, MN) with a sample rate of $128 \mathrm{~Hz}$. Cardiac conduction and rhythm disturbances were automatically analyzed by a Holter-ECG system (Danica Holter Replay Unit; Danica Biomedical, Borlänge, Sweden). The classification made by the Holter system was used to distinguish normal and non-normal QRS complexes. The Holter system uses a classification of the QRS morphology to distinguish among ventricular ectopic beats, signal noise, and normal complexes. The consecutive RR intervals and the corresponding classification code were exported to an ASCII text file.

For the frequency domain analysis, $5 \mathrm{~min}$ epochs of data were analyzed by custom-made software (30). The time series of RR intervals were re-sampled at a frequency of two samples/second $(2 \mathrm{~Hz})$. Gaps in the time series owing to non-normal RR intervals (QRS-labeled by the Aspect System classification as noise or ectopic beats) were filled with values calculated by linear interpolation between the adjacent normal RR intervals. The computer program checked for misclassified drop beats deviating more than three SD from the mean normal RR interval of each epoch. Epochs with more than $4 \%$ of non-normal RR intervals were excluded from further analysis. At least $50 \%$ of a recording had to be acceptable to be included, in accordance with issued guidance (Task Force of the European Society of Cardiology and the North American Society of Pacing and Electrophysiology, 1996; ref. 6). The frequency domain of the time series of RR intervals was analyzed with an auto-regression method (31). The model order and number of coefficients in the polynomial describing the time series was constantly (same model order for all epochs) set to autoregression $=18$. The mean $R R$ interval of each time series was subtracted and then de-trended by applying linear regression. The power spectrum of the frequency domains was divided into four different frequency bands: Tot Pow: $0.0033-0.40 \mathrm{~Hz}\left(\mathrm{~ms}^{2}\right)$; VLF Pow: $0.0033-$ $0.04 \mathrm{~Hz}\left(\mathrm{~ms}^{2}\right)$; LF Pow: $0.04-0.15 \mathrm{~Hz}\left(\mathrm{~ms}^{2}\right)$; and HF Pow: $0.15-0.40$ $\mathrm{Hz}\left(\mathrm{ms}^{2}\right)$. In the time domain, the mean of the SD of all normal RR intervals for all 5 min segments of each ECG recording (SDNN index) was calculated.

\section{Statistics}

Data are presented as numbers, percentage, mean values with $1 \mathrm{SD}$ or confidence interval, or medians with quartiles and minimum and maximum values as indicated. Analyses were computed in JMP software package, version 8.0.1 (SAS Institute, Cary, NC) using nonparametric Kruskal-Wallis test because our data could not be adequately modeled by a normal distribution (Supplementary Data online).

A $P$ value of $<0.05$ was defined as significant. One patient from the PT group showed values above the +3 SDs from the mean of the components frequencies and was therefore excluded from further statistical testing.

A series of stepwise multiple regression was performed in order to identify variables influencing HRV parameters (gender, age at study, BMI at study, maternal hypertension, maternal diabetes, and prenatal steroid administration).

The independent variables with F-to-enter $=4$ were entered into logistic analysis models.

\section{SUPPLEMENTARY MATERIAL}

Supplementary material is linked to the online version of the paper at http:// www.nature.com/pr.

\section{STATEMENT OF FINANCIAL SUPPORT}

This work was supported by the Samariten Foundation (Stockholm, Sweden), Freemasons Foundation (Stockholm, Sweden), and Sällskapet Barnavård (Stockholm, Sweden).

Disclosure: We have no potential conflict of interest relevant to this article.

\section{REFERENCES}

1. Barker DJ, Osmond C, Golding J, Kuh D, Wadsworth ME. Growth in utero, blood pressure in childhood and adult life, and mortality from cardiovascular disease. BMJ 1989;298:564-7.

2. Kajantie E, Osmond C, Barker DJ, Eriksson JG. Preterm birth—a risk factor for type 2 diabetes? The Helsinki birth cohort study. Diabetes Care 2010;33:2623-5. 
3. Strauss RS. Adult functional outcome of those born small for gestational age: twenty-six-year follow-up of the 1970 British Birth Cohort. JAMA 2000;283:625-32.

4. Godfrey KM, Barker DJ, Osmond C. Disproportionate fetal growth and raised IgE concentration in adult life. Clin Exp Allergy 1994;24:641-8.

5. Galland BC, Taylor BJ, Bolton DP, Sayers RM. Heart rate variability and cardiac reflexes in small for gestational age infants. J Appl Physiol 2006;100:933-9.

6. Malik M. Heart rate variability: standards of measurement, physiological interpretation and clinical use. Task Force of the European Society of Cardiology and the North American Society of Pacing and Electrophysiology. Circulation 1996;93:1043-65.

7. Bigger JT, Fleiss JL, Rolnitzky LM, Steinman RC. The ability of several short-term measures of RR variability to predict mortality after myocardial infarction. Circulation 1993;88:927-34.

8. Bigger JT Jr, Fleiss JL, Steinman RC, Rolnitzky LM, Kleiger RE, Rottman JN. Frequency domain measures of heart period variability and mortality after myocardial infarction. Circulation 1992;85:164-71.

9. Kleiger RE, Miller JP, Bigger JT Jr, Moss AJ. Decreased heart rate variability and its association with increased mortality after acute myocardial infarction. Am J Cardiol 1987;59:256-62.

10. Dekker JM, Schouten EG, Klootwijk P, Pool J, Swenne CA, Kromhout D. Heart rate variability from short electrocardiographic recordings predicts mortality from all causes in middle-aged and elderly men. The Zutphen Study. Am J Epidemiol 1997;145:899-908.

11. Kobayashi F, Watanabe T, Akamatsu Y, et al. Acute effects of cigarette smoking on the heart rate variability of taxi drivers during work. Scand J Work Environ Health 2005;31:360-6.

12. Browne CA, Colditz PB, Dunster KR. Infant autonomic function is altered by maternal smoking during pregnancy. Early Hum Dev 2000;59:209-18.

13. Rodríguez-Colón SM, Bixler EO, Li X, Vgontzas AN, Liao D. Obesity is associated with impaired cardiac autonomic modulation in children. Int $\mathrm{J}$ Pediatr Obes 2011;6:128-34.

14. Johansson S, Norman M, Legnevall L, Dalmaz Y, Lagercrantz H, Vanpée $\mathrm{M}$. Increased catecholamines and heart rate in children with low birth weight: perinatal contributions to sympathoadrenal overactivity. J Intern Med 2007;261:480-7.

15. Aziz W, Schlindwein FS, Wailoo M, Biala T, Rocha FC. Heart rate variability analysis of normal and growth restricted children. Clin Auton Res 2012;22:91-7.

16. De Rogalski Landrot I, Roche F, Pichot V, et al. Autonomic nervous system activity in premature and full-term infants from theoretical term to 7 years. Auton Neurosci 2007;136:105-9.
17. Chatow U, Davidson S, Reichman BL, Akselrod S. Development and maturation of the autonomic nervous system in premature and full-term infants using spectral analysis of heart rate fluctuations. Pediatr Res 1995;37:294302.

18. Khattak AZ, Padhye NS, Williams AL, Lasky RE, Moya FR, Verklan MT. Longitudinal assessment of heart rate variability in very low birth weight infants during their NICU stay. Early Hum Dev 2007;83: 361-6.

19. Patural H, Barthelemy JC, Pichot V, et al. Birth prematurity determines prolonged autonomic nervous system immaturity. Clin Auton Res 2004;14:391-5.

20. Assali NS, Brinkman CR 3rd, Woods JR Jr, Dandavino A, Nuwayhid B. Development of neurohumoral control of fetal, neonatal, and adult cardiovascular functions. Am J Obstet Gynecol 1977;129:748-59.

21. Boguszewski MC, Johannsson G, Fortes LC, Sverrisdóttir YB. Low birth size and final height predict high sympathetic nerve activity in adulthood. J Hypertens 2004;22:1157-63.

22. Neovius M, Janson A, Rössner S. Prevalence of obesity in Sweden. Obes Rev 2006;7:1-3.

23. Bjelakovic B, Ilic S, Dimitrijevic L, et al. Heart rate variability in infants with central coordination disturbance. Early Hum Dev 2010;86: $77-81$.

24. Højgaard MV, Holstein-Rathlou NH, Agner E, Kanters JK. Dynamics of spectral components of heart rate variability during changes in autonomic balance. Am J Physiol 1998;275(1 Pt 2):H213-9.

25. Høgberg U, Larsson N. Early dating by ultrasound and perinatal outcome. A cohort study. Acta Obstet Gynecol Scand 1997;76:907-12.

26. Marsál K, Persson PH, Larsen T, Lilja H, Selbing A, Sultan B. Intrauterine growth curves based on ultrasonically estimated foetal weights. Acta Paediatr 1996;85:843-8.

27. Morris SS, Victora CG, Barros FC, et al. Length and ponderal index at birth: associations with mortality, hospitalizations, development and postnatal growth in Brazilian infants. Int J Epidemiol 1998;27:242-7.

28. Cole TJ, Bellizzi MC, Flegal KM, Dietz WH. Establishing a standard definition for child overweight and obesity worldwide: international survey. BMJ 2000;320:1240-3.

29. Marshall WA, Tanner JM. Variations in pattern of pubertal changes in girls. Arch Dis Child 1969;44:291-303.

30. Storck N, Ericson M, Lindblad L, Jensen-Urstad M. Automatic computerized analysis of heart rate variability with digital filtering of ectopic beats. Clin Physiol 2001;21:15-24.

31. Kay SM, Marple SL. Spectrum analysis:a modern perspective. Proc IEEE 1981;69:1380-419. 